\title{
Anabases
}

ANABASES Traditions et réceptions de l'Antiquité

18 | 2013

Varia

\section{Hippolyte Tautain. Origine littéraire et identité sémantique d'un personnage de Madame Bovary}

Michael Paschalis

\section{OpenEdition}

Journals

Édition électronique

URL : http://journals.openedition.org/anabases/4404

DOI : 10.4000/anabases.4404

ISSN : 2256-9421

Éditeur

E.R.A.S.M.E.

Édition imprimée

Date de publication : 1 octobre 2013

Pagination : 85-108

ISSN : 1774-4296

\section{Référence électronique}

Michael Paschalis, « Hippolyte Tautain. Origine littéraire et identité sémantique d'un personnage de Madame Bovary ", Anabases [En ligne], 18 | 2013, mis en ligne le 01 novembre 2016, consulté le 14 novembre 2019. URL : http://journals.openedition.org/anabases/4404 ; DOI : 10.4000/anabases.4404 


\section{Hippolyte Tautain Origine littéraire et identité sémantique d'un personnage de Madame Bovary}

Michael Paschalis

Quelle est l'origine littéraire d'Hippolyte Tautain, du garçon d'écurie boiteux qui apparait dans le roman de Flaubert, Madame Bovary? Le lecteur se souvient de sa douleur horrible lors de l'opération ratée que Charles Bovary tente à son pied déformé et qu'elle aboutit forcément à une amputation. Certains commentateurs ont déjà observé des liens entre Hippolyte et Hippolytos, le héros d'Euripide dans la tragédie du même nom et celui de la Phèdre de Sénèque et de Racine. Dans cette étude, je propose de mettre en valeur, à travers le texte et les manuscrits de Flaubert, la voie par laquelle Flaubert est arrivé au mythe de ce héros, la dimension intertextuelle de ce rapport, l'aspect sémantique des noms « Hippolytos » et « Hippolyte » dans leur évolution, ainsi que certains éléments qui concernent l'identité romancière du héros de Flaubert ${ }^{1}$.

1 Une première version de cet article a paru en grec moderne dans la revue Nea Hestia, dédiée à la célébration des cent-trente ans de la mort de Gustave Flaubert (Décembre 2010). J'utilise le nom " Hippolyte " pour le héros de Flaubert et " Hippolytos " pour le héros de la mythologie grecque. Pour les manuscrits de Mme de Bovary, j'ai consulté le site de l'université de Rouen (http://flaubert.univ-rouen.fr). Je voudrais remercier ma collègue Mélina Tamiolaki pour avoir traduit mon article en français. 


\section{Remarques sur l'origine littéraire d'Hippolyte}

Flaubert choisissait soigneusement les noms des caractères de ses romans ${ }^{2}$. É. Zola, dans une lettre bien connue, dans laquelle il discute les noms propres chez ses contemporains romanciers, commente l'importance des noms chez Flaubert : "Flaubert poussait ainsi la religion du nom jusqu'à dire que, le nom n'existant plus, le roman n'existait plus ${ }^{3}$."

Madame Bovary en constitue un exemple caractéristique. Flaubert ne donne pas d'indications précises sur l'étymologie des noms de son roman ; néanmoins, dans un manuscrit, il commente le nom du pharmacien "Homais " qu'il lie avec le mot latin homo (= homme) : "Homais vient de l'Homo ${ }^{4}$. " La plupart des noms chez Flaubert sont " transparents ${ }^{5}$ " d'un point de vue sémantique. L'usage ironique des noms est prédominant ; il se fonde sur les contrastes qu'il implique et fonctionne souvent comme une parodie du romantisme. Par exemple, le premier amant d'Emma porte le prénom romantique "Rodolphe ", mais son nom est "Boulanger ». Deux héros antiromantiques du roman portent des noms qui renvoient au bonheur : le marchand et usurier "Lheureux » (l'heureux) qui détruit Emma, et la servante d'Emma " Félicité ». Une autre invention littéraire réussie de Flaubert est le nom du char qui fait le trajet Yonville-Rouen : il s'appelle " Hirondelle », mais le nom de son conducteur, " Hivert », renvoie à l'hiver.

Les commentateurs ont constaté la présence abondante des noms qui renvoient aux animaux et plus spécifiquement aux bovins. L'amplification du champ sémantique des bovins dans la langue et dans le récit du roman est si grande, que J. Culler a commenté ce phénomène avec un jeu de mots : "We might conclude that is not a realist novel so much as a vealist novel ${ }^{6}$. " À cette catégorie appartiennent tout d'abord les noms "Bovary " (lat. bo(v)arius, provenant de bos) et "Charbovari " (Charles Bovary), que Charles lui-même utilise pour se présenter à ses camarades et qui évoque "le char aux bœufs ". Sa première épouse s'appelait " Héloise Dubuc ", le prénom étant romantique et le nom évoquant le bouc. Notons aussi que l'adjectif latin $b o(v)$ arius, d'où provient le nom "Bovary ", était utilisé en Rome antique pour dénoter le marché des bœufs (forum boarium). L'épouse de "Léon " (leo = lion,

2 Voir D.L. Demorest, L'expression figurée dans l'ouvre de Flaubert, Paris, 1931, p. 471-472; J. Pommier, "Noms et prénoms dans Madame Bovary", Mercure de France 306 (1949), p. 244-264 ; L. PARKS, "Flaubert's Name Game in Madame Bovary ", The South Central Bulletin 31 (1971), p. 207-210 ; J. Culler, "The Uses of Madame Bovary ", Diacritics 11 (1981), p. 74-83 ; L.M. Porter, E.F. Gray, Gustave Flaubert's Madame Bovary : A Reference Guide, Westport, Connecticut / London, 2002, p. 103-105.

3 É. Zola, Correspondances: Les lettres et les arts, Paris, 1908, p. 196.

4 Plans et scénarios, folio 46v.

5 Pour ce terme, voir infra (unité 3).

6 Culler, "The Uses ", p. 78. 
mais Léon est lâche !) porte le nom "Lebœuf ", mais son prénom est romantique : "Léocadie » (Leucadia). Enfin, le nom du maire «Tuvache » renvoie à la vache (deux vaches ou tu es une vache $)^{7}$. Les héros du roman inachevé de Flaubert, Bouvard et Pécuchet, sont aussi liés aux bovins et aux troupeaux ${ }^{8}$.

En ce qui concerne l'invention du nom "Hippolyte », des interprétations diverses ont été avancées. La plus ancienne est probablement celle de D. L. Demorest, qui considère que le prénom évoque la marche sautante du héros boiteux : « Le pied-bot qui en marchant saute comme son nom ${ }^{9}$. "Selon J. Pommier, d'autre part, Flaubert a emprunté ce nom au traité médical de Vincent Duval sur le pied-bot ${ }^{10}$, dans lequel apparât un patient qui s'appelle Pierre Hippolyte Vincent ${ }^{11}$. En revanche, d'après M. D. Palmer, Flaubert a été inspiré par le roman Histoire d'Hypolite, comte de Duglas de Mme d'Aulnoy (Mme d'Aulnoy = Marie-Catherine, comtesse d'Aulnoy), paru en $1690^{12}$. Les récits de Mme d'Aulnoy constituaient une lecture bien aimée de Flaubert, quand il était un enfant, mais l'Histoire d'Hypolite n'appartient pas à ces lectures ${ }^{13}$. Palmer estime que le public de l'époque de Flaubert " aurait reconnu qu'Hippolyte représentait la dégénérescence complète du héros romantique qui occupait une place importante dans la production romancière populaire française pour plus de deux siècles ». Il constate en plus une liaison principale avec le héros tragique de la Phèdre de Racine, qui, selon son point de vue, "renforce la dimension ironique, anti-romantique du roman ", mais ne fournit pas de précisions supplémentaires sur ce point ${ }^{14}$. Tilby, enfin, fait aussi une référence courte à une liaison probable du nom " Hippolyte " avec la tragédie ancienne et à un usage ironique du nom ${ }^{15}$.

7 PARKS, «Flaubert's Name Game », p. 207.

8 H. Levin, The Gates of Horn: A Study of Five French Realists, New York, 1963, p. 249. Levin mentionne les mots latins bos et peculpecus.

9 L'expression figurée, p. 472.

10 Voir aussi, infra (unité 4).

11 «Noms et prénoms ", p. 260-261.

12 Pour une édition récente de ce roman, voir S.J. DAY, Madame d'Aulnoy, Histoire d'Hypolite, comte de Duglas, Sommerset, 1994.

13 Voir le lemme et la note relative qui existe sur la copie de la bibliothèque restituée de Flaubert (http://flaubert.univ-rouen.fr/bibliotheque/).

14 M.D. Palmer, "The Literary Ancestry of Flaubert's Hippolyte ", Studies in the Novel 3 (1971), p. 97-98.

15 T. Unwin, The Cambridge Companion to Flaubert, Cambridge, 2004, p. 22. 
L'étude de Margaret Church ${ }^{16}$ et la biographie importante de Flaubert publiée récemment par Frederick Brown ${ }^{17}$ comportent la discussion la plus détaillée sur l'origine littéraire d'Hippolyte. F. Brown répète certains des arguments de Church (sans citer son œuvre) et en ajoute d'autres; il donne ainsi une dimension nouvelle à l'interprétation principalement symboliste de la chercheuse américaine. Il remarque, entre autres, que, parmi les animaux, Flaubert avait une préférence pour le cheval, parce qu'il sentait avec cet animal " une identification profonde ${ }^{18}$ » en raison des expériences personnelles qui étaient empreintes dans sa mémoire. Il cite la correspondance de Flaubert pour prouver cette identification et en général la présence importante du cheval en tant que métaphore littéraire et critique chez Flaubert. En ce qui concerne Madame Bovary, F. Brown remarque que le cheval est lié à des moments importants de la vie d'Emma, dont le plus important est « l'opération à laquelle elle soumet le garçon boiteux, en espérant qu'un grand succès médical aurait réussi là où l'idylle a raté et qu'il l'aurait libérée de la prison de la province ». Le biographe continue ainsi :

"Flaubert aimait la liaison ironique de la mythologie grecque avec les paysans de Normandie et avait des raisons sérieuses pour donner à Hippolyte le nom du fils de Thésée. Le garçon d'écurie est, à sa manière, tout comme le prince grec, un athlète pur qui est détruit par une femme endiablée. L'un a une faiblesse pour les chevaux, l'autre est une forme de Centaure. Hippolytos est un jeune vertueux qui suscite une passion incestueuse, tandis qu'Hippolyte est un monstre de nature ; et pourtant, sa laideur dispose des vertus que son entourage et surtout Emma ne peuvent pas apprécier [...]. Tous ont investi personnellement sur le succès ou l'échec de l'opération ${ }^{19}$."

La comparaison entre Hippolyte et Hippolytos qu'entreprend Brown est à mon avis la plus radicale, bien que sa perspective soit idéalisée et que l'auteur crée, en plus, l'impression qu'Hippolytos est le fils de Phèdre ${ }^{20}$. Il apparât que le biographe de Flaubert a travaillé sur un sommaire du mythe de Phèdre et d'Hippolytos.

Une question étroitement liée à cette problématique concerne le texte du mythe d'Hippolytos que le lecteur devrait prendre en considération lorsqu'il compare

16 M. Church, Structure and Theme: Don Quixote to James Joyce, Columbus, 1983, p. 73-75. Cf. aussi, F. EMPTAZ, " Gustave Flaubert apprenti orthopédiste : de la bibliothèque paternelle à l'espace Romanesque ", in Y. LECLERC (sous la dir.), La bibliothèque de Flaubert: inventaires et critiques, Rouen, 2001, p. 221-235, qui met l'accent sur Hippolyte, mais dans la perspective d'Emma.

17 F. Brown, Flaubert: A Biography, New York, 2006, p. 307-309.

18 Ibid., p. 307.

19 Ibid., p. 308-309.

20 Hippolytos est le fils de l'Amazone Antiope ou Hippolyte. Les tragédies qui traitent ce sujet prennent soin de souligner au moment crucial de l'expression de la passion de Phèdre que le jeune homme est né d'une autre mère. Voir par exemple, Euripide, Hippolyte 306-309 ; Sénèque, Phèdre 232 ; Racine, Phèdre 201-205. 
Hippolyte et Hippolytos. Pour les Français, le texte classique qui décrit la mort d'Hippolyte était bien sûr la Phèdre de Racine et plus précisément les paroles de Théramène à la scène VI de l'acte V (1498-1593), qui commence avec le vers bien connu : "À peine nous sortions des portes de Trézène. " Flaubert mentionne un vers de la Phèdre dans ses notes sur son voyage en Grèce ( reste impur des brigands dont j’ai purgé la terre ", 1046) ${ }^{21}$. Un autre passage de la Phèdre qu'on rencontre dans un manuscrit de Madame Bovary et que Flaubert a ensuite supprimé est significatif. Dans le chapitre VI de la deuxième partie, Emma visite l'abbé Bournisien à l'église et tente de lui ouvrir son cœur. Quand elle lui dit qu'elle est mal et qu'elle souffre, celui-ci pense qu'il s'agit d'une souffrance corporelle. Dans une version plus ancienne de cette scène ${ }^{22}$, Emma précisait : "Mon mal vient de plus loin. " Avec cette même phrase qui est aussi bien connue, Phèdre commence à avouer à sa nourrice CEnone l'amour torturant qu'elle éprouve pour son beau-fils Hippolytos. La relation intertextuelle entre Madame Bovary et la Phèdre de Racine est donc évidente.

Or, mon étude prend en plus comme point de départ le fait que Flaubert devait aussi avoir connaissance des modèles anciens de cette tragédie, à savoir l'Hippolytos d'Euripide et la Phèdre de Sénèque, même s'il ne les mentionne pas. Cela peut être supposé, non seulement à cause de son étude passionnée de la littérature ancienne qui est bien connue, mais aussi pour une raison supplémentaire : parce que les éditions de la Phèdre de Racine, antérieures ou contemporaines de Flaubert, comportaient des références courtes ou détaillées aux modèles de la tragédie ancienne et citaient dans la préface ou dans les commentaires les passages parallèles d'Euripide et de Sénèque ${ }^{23}$. En plus, certaines d'entre elles reproduisaient la préface éclairante que Racine lui-même a écrite pour sa tragédie ${ }^{24}$. En me fondant sur une analyse sémantique, j’avance la thèse selon laquelle Flaubert n'aurait pas pu élaborer les significations du nom « Hippolyte " au cours du récit sans avoir eu une connaissance non seulement de l'étymologie littéraire de l'« Hippolytos " mythique mais aussi du texte même de l'Hippolytos d'Euripide et de la Phèdre de Sénèque - Racine n'est pas utile en ce regard.

21 J. Bruneau, G. Flaubert, Euvres complètes, Paris, 1964, t. 2, p. 668.

22 Brouillons, vol. 2, folio 263v.

23 Cf. aussi, infra.

24 Pour cette préface, voir par exemple l'édition Euvres de Jean Racine, Nouvelle édition, plus correcte et plus ample que les précédentes, Tome second, Paris, Les Libraires Associés, 1786, qui est incluse dans le catalogue de la bibliothèque restituée de Flaubert, tel qu'il apparaît sur le site de l'université de Rouen. 


\section{« La mort d'Hippolyte [...]. Tout le monde devrait savoir ce morceau par cœur" (Flaubert)}

Au lemme "Hippolyte " du Dictionnaire des idées reçues, Flaubert note : "La mort d'Hippolyte, le plus beau sujet de narration que l'on puisse donner. Tout le monde devrait savoir ce morceau par cœur ${ }^{25}$." Dans la préface qu'il préparait pour le Dictionnaire Flaubert écrit entre autres que le sujet de chaque lemme concernant la littérature devrait être complètement banal et stéréotypé : "Il faut donc honnir toute espèce d'originalité comme dangereuse, sotte [...] ». Ces paroles apparaissent dans une lettre adressée à Louise Colet le 16 décembre 1852, c'est-à-dire dans une période pendant laquelle l'auteur travaillait sur Madame Bovary (1851-1856) ${ }^{26}$. Dans cette unité de mon étude, je vais montrer que le contenu du lemme sur Hippolyte du Dictionnaire des idées reçues était présent à l'esprit de Flaubert lors de l'écriture de Madame Bovary et plus précisément lors du dessin des premiers chapitres du roman. Je vais aussi avancer l'idée d'un rapport génétique que ce lemme entretient avec la conception et la formation du héros du roman qui porte le même nom.

Au chapitre III de la première partie de Madame Bovary Charles se prépare timidement à demander au père d'Emma sa fille en mariage. Celui-ci prévient son intention et lui dit qu'il est d'accord avec son vœu apparent; mais il précise ensuite qu'il doit d'abord demander l'avis d'Emma et ajoute que le signe qu'il va lui envoyer dans le cas d'une réponse positive de sa part, sera de pousser " tout grand l'auvent de la fenêtre contre le mur ". Flaubert continue ainsi : "Charles attacha son cheval à un arbre. Il courut se mettre dans le sentier ; il attendit. Une demi-heure se passa, puis il compta dix-neuf minutes à sa montre ${ }^{27}$."

D'après les versions précédentes de ce passage, Charles fait aussi d'autres choses pour passer le temps d'attente, jusqu'à ce que l'auvent de la fenêtre s'ouvre : il essaie de compter jusqu'à mille, il regarde les aiguilles de sa montre, il va voir si son cheval est détaché, etc. Une autre activité présente aussi un intérêt particulier ; elle apparaît dans

25 Flaubert, Dictionnaire des idées reçues, s.v.

26 J. Bruneau, G. Flaubert, Correspondance, t. 2 (juillet 1851-décembre 1858), Paris, 1980, p. 207-210 (lettre à Louise Colet). Cf. aussi, PORTer et Gray, Gustave Flaubert's Madame Bovary, p. 83-85. Le Dictionnaire a finalement paru presque trente ans après la mort de Flaubert, d'après les notes qu'il avait laissées. Selon une interprétation, il serait destiné à constituer la suite du roman Bouvard et Pécuchet, que l'auteur n'a pas achevé. Néanmoins, cette idée le hantait depuis longtemps, dès sa jeunesse, il l'élaborait constamment pour plusieurs années et avait rassemblé une partie de son matériel bien avant les années 1870 . Le dessin et le titre de l'édition sont déjà connus de Louis Bouilhet en septembre 1850 et des lemmes potentiels du Dictionnaire apparaissent dans la première version de l'Éducation sentimentale (1845) et dans Madame Bovary.

27 Flaubert, Euvres complètes, t. 1, p. 582. 
trois manuscrits du roman. Je cite le passage en sa forme finale, avant que Flaubert le supprime : "Pour que le temps lui parut moins long, il essaya [...] de se rappeler le commencement de la mort d'Hippolyte qu'il savait par $\operatorname{cour}^{28}$. " Charles essaie de se souvenir ("pour réciter", selon certains manuscrits) de la mort d'Hippolyte " qu'il savait par cour ", tout comme le recommande le Dictionnaire des idées reçues ("Tout le monde devrait savoir ce morceau par cœur »). Mais son embarras est si grand, qu'il a oublié même le début de ce stéréotype littéraire absolu !

D'après les multiples versions du mythe, Phèdre est l'épouse du héros Thésée et est possédée par une passion amoureuse pour son beau-fils Hippolytos, fils de l'Ama-

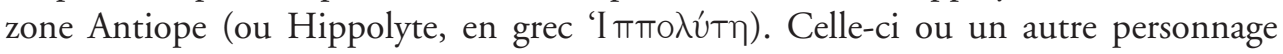
calomnie Hippolytos, en disant à Thésée que le jeune homme a violé ou a tenté de violer sa belle-mère. Thésée le maudit, en réalisant un vœu que le dieu Poséidon lui avait accordé ; un peu plus tard le dieu envoie de la mer un taureau monstrueux; les chevaux du héros s'emballent et le tirent sur les rochers en déchirant son corps. Phèdre, consciente de sa culpabilité, se suicide avant ou après la fin tragique d'Hippolytos.

Emma Bovary est aussi une femme mariée avec des rapports extraconjugaux qui est amenée au suicide. Il semble donc que la passion de l'héroïne ancienne ne soit pas passée inaperçue pour Flaubert, quand il dressait le portrait psychologique d'Emma. Il y a aussi un autre élément qui lie Emma et Phèdre. Comme nous l'avons noté auparavant, la fortune d'Hippolyte, le garçon d'écurie boiteux, évoque le héros tragique Hippolytos. Mais cette liaison est effectuée en des termes qui constituent une mise en question directe du récit banal de la mort d'Hippolytos, sur laquelle Flaubert ironise dans le Dictionnaire des idées reçues et que Charles connait par coeur mais dont il n'arrive pas à se souvenir. Dans le chapitre XI de la deuxième partie, Charles fait une opération sur le pied déformé d'Hippolyte. L'idée de l'opération provient du pharmacien Homais qui a entendu parler d'une méthode nouvelle de guérison pour les pieds bots et a donc voulu en faire usage pour augmenter sa propre réputation. Celui-ci a convaincu Emma qui, par la suite, a conçu cette opération comme une occasion pour l'ascension sociale de son mari et pour sa libération personnelle. Charles qui lui fait toujours plaisir a cédé. Le malheureux Hippolyte a subi tant de pressions qu'il a accepté enfin de se faire opérer le pied. L'opération est survenue sous la pression et les attentes de la communauté entière. Emma, dans la mesure où elle persuade Charles de procéder à l'opération ratée du pied d'Hippolyte, évoque, mutatis mutandis, le rôle de Phèdre dans la destruction du héros mythique Hippolytos.

La gangrène s'étend rapidement à la jambe opérée d'Hippolyte et sa vie est sauvée par le médecin Canivet qui est convoqué de la ville de Neufchâtel et procède à une amputation. Lorsque Canivet se prépare à amputer Hippolyte, Charles pense que «si

La demande en mariage - définitif, folio 53. 
Hippolyte plus tard venait à mourir, c'est lui qui l'aurait assassiné29 " et en imagine les conséquences, à savoir la diffamation, l'opprobre et la catastrophe.

Finalement, l'opération ratée que Charles a tentée au pied d'Hippolyte n'a pas provoqué sa mort ; le même personnage, dans une version antérieure, n’a pas réussi à se souvenir de la mort d'Hippolytos pour la réciter, quand il attendait avec impatience la réaction d'Emma à sa proposition de mariage. Les deux séries d'événements font partie de la réponse de Flaubert au stéréotype littéraire de la mort d'Hippolytos et à la conception sous-jacente du Dictionnaire qui " honnissait toute espèce d'originalité " dans le traitement d'un sujet. Le fait que Charles n'a pas pu se rappeler ce que tous les gens cultivés devraient savoir par cœur constitue au premier abord un commentaire ironique sur sa panique et probablement sur sa scolarité difficile. Mais la fonction la plus importante de cette scène est qu'elle annule au niveau programmatique la mort d'Hippolytos et cela a un rapport étroit avec la fortune d'Hippolyte dans le roman. Hippolyte ne meurt pas, ce qui est dû au fait que dans la suite du récit, autant pendant l'opération que plus tard, son handicap acquiert une importance plus grande que sa mort éventuelle : il évoque, entre autres choses, l'incompétence et la recherche de l'intérêt personnel qui caractérisent ceux qui sont responsables de sa situation.

\section{Hippolytos et Hippolyte : la dynamique sémantique des noms}

"Charles attacha son cheval à un arbre... il essaya... réciter la mort d'Hippolyte qu'il savait par coeur [...] il alla voir si son cheval n'était pas détaché $e^{30}$. "

Dans un des manuscrits de Madame Bovary ${ }^{31}$ la période d'attente de Charles est entourée par des références à son cheval : il l'" attache " tout d'abord à un arbre, un peu plus tard il va voir si son cheval «n'était pas détaché ». La première référence est présente dans tous les manuscrits, ainsi que dans la version définitive du texte. Flaubert a supprimé la deuxième pour des raisons narratologiques, mais cela ne réduit pas son importance dans une étude sémantique. En effet, les phrases " attacha son cheval ", " la mort d'Hippolyte " et "si son cheval n'était pas détaché " forment une combinatoire sémantique (semantic cluster). La combinatoire sémantique est constituée par des unités sémantiques simples ou composées qui sont liées entre elles de manières diverses (rapports de synonymie, d'antonymie, d'hyponymie, etc.). Les noms propres font partie de l'ensemble sémantique et coopèrent avec les autres unités. Hors du texte,

29 Euvres complètes, t. 1, p. 636.

30 Dans l'édition du manuscrit la phrase " réciter [...] par cœur " apparaît en italiques comme un ajout de l'auteur.

31 Brouillons, vol. 1, folio $117 \mathrm{v}$. 
certains noms, simples ou composés, sont " transparents » d'un point de vue sémantique, tandis que d'autres sont " opaques", parce que leur sens est obscur ou parce que leur morphologie n'explique pas leur composition. Néanmoins, le sens qu'on reconnaît aux noms hors du texte et leur dimension grammaticale et lexicographique n'ont pas de valeur contraignante et n'anticipent pas leur fonction dans le cadre du récit. En bref, dans un contexte narratif, les noms " libèrent " les sens qu'ils comportent, en développent d'autres et évoluent au cours du récit; en revanche, leurs composants peuvent fonctionner indépendamment l'un de l'autre. Les noms " transparents », réels ou fictifs, sont plus faciles à interpréter ; les noms " opaques ", au contraire, créent des difficultés au lecteur, mais ils lui donnent aussi souvent une liberté plus grande d'interprétation. En général, les noms sont des unités linguistiques qui disposent d'une dynamique extraordinaire : ils peuvent impliquer des descriptions condensées ou de courts récits et suggèrent au lecteur plusieurs voies d'interprétation ${ }^{32}$.

Dans la combinatoire sémantique citée plus haut, les phrases " attacha son cheval » et "si son cheval n'était pas détaché » entourent la phrase "la mort d'Hippolyte ». Elles évoquent ainsi l'étymologie du nom ancien transparent " Hippolytos », qui dérive du substantif ímாоऽ (cheval) et du verbe $\lambda \hat{v} \omega$ (détacher). Il y a une discussion sur le sens original des composants du nom "Hippolytos ${ }^{33}$ " que, selon le mythe, le

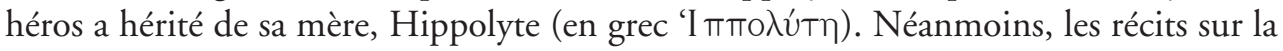
fin tragique du héros suggèrent une étymologie différente : " celui qui a été démembré par ses chevaux " (verbe grec $(\delta\llcorner\alpha) \lambda u ́ \omega=$ détruire, dissoudre, démembrer). Cette étymologie apparait pour la première fois dans la combinatoire sémantique d'un court récit de Virgile : namque ferunt fama Hippolytum [...] turbatis distractus equis ${ }^{34}$. Une variation est aussi présente chez Ovide ( hic latet Hippolytus loris direptus equorum ${ }^{35}$ ), ainsi que chez Sénèque (per illa qua distractus Hippolytus loca ${ }^{36}$ ). L'étymologie apparaît de nouveau dans le martyre de Saint Hippolyte, qui, d'après le verdict du juge, est entraîné par deux chevaux, ses jambes étant attachées, tout comme le héros mythique, de sorte que sa manière de mourir confirme son nom : ergo [...] Hippolytus [...] / intereatque feris dilaceratus equis ${ }^{37}$.

32 Voir à ce propos M. Paschalis, Virgil's Aeneid: Semantic Relations and Proper Names, Oxford, 1997, p. 1-33.

33 Voir R. Halleran, Euripides, Hippolytus, Oxford, 1984, p. 21 ; L. Dubois, « Hippolytos

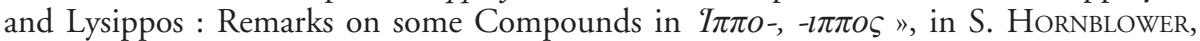
E. MatThews (éd.), Greek Personal Names : Their Value as Evidence, Oxford, 2000, p. $41-52$.

34 Virgile, Énéide 7, 765-767 «Selon la légende, Hippolyte [...] écartelé par des chevaux affolés ".

35 Ovide, Fasti 3, 265 "C'est là qu'Hippolyte a disparu, déchiré par ses coursiers furieux ".

36 Sénèque, Phèdre 1106 "par ces lieux où Hippolyte a été déchiré".

37 Prudentius, Ad Valerianum Episcopum de passione Hippolyti beatissimi martyris, 87-88 "qu'Hippolyte meure démembré par ces chevaux ». Le récit de Théramène dans la Phèdre 
Dans le passage de Flaubert cité plus haut le nom " Hippolyte » est entouré par les verbes "attacher » et « détacher ». Comme nous l'avons noté auparavant, les unités sémantiques d'un ensemble peuvent être liées entre elles, selon le cas ou simultanément, de manières diverses : rapports de synonymie, d'antonymie ou d'hyponymie. Je vais proposer dans la suite de mon analyse que la liaison du composant $\lambda \dot{\omega} \omega$ du nom d'Hippolyte avec des sens synonymes et opposés a une importance cruciale pour l'interprétation du nom chez Madame Bovary, qui semble sur ce point avoir hérité l'usage du nom de l'Antiquité. Déjà le récit de la mort d'Hippolytos dans la tragédie d'Euripide du même nom met en valeur le deuxième composant du nom en jouant avec les sens d'" attacher " et de " détacher " : le corps du héros est " enroulé » entre les rênes des chevaux, de sorte qu'il est entraîné et cassé sur les rochers ; ensuite Hippolytos " est libéré » des rênes et tombe sur terre demi-mort :

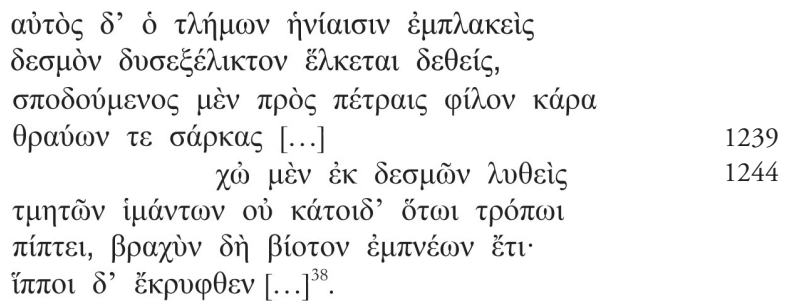

Nous avons déjà observé que les noms, dès leur insertion dans un récit, acquièrent une fonction dynamique : ils « libèrent » les sens présents, ils en développent d'autres, ils " corrigent " les sens antérieurs et enfin ils évoluent au cours de la narration. Un exemple caractéristique est la Phèdre de Sénèque, une pièce dans laquelle le nom " Hippolytus » a développé plusieurs sens, indépendants du récit mythique de la mort du héros ${ }^{39}$. Le nom Hippolyte a donc été inséré dans Madame Bovary en portant en soi un passé mythique, une production littéraire créative, ainsi qu'une dynamique inhérente qui consiste en ses composants.

Flaubert introduit Hippolyte au début de la deuxième partie du roman, en suivant une stratégie narrative concrète : soit il ne mentionne pas son nom, soit il l'introduit de manière anonyme. Son nom complet apparaît dans le chapitre VIII de la deuxième partie dans un contexte précis que je vais commenter dans la suite de mon analyse.

de Racine n'évoque pas en premier abord un sens concret du nom, mais cela est en rapport avec les priorités littéraires de l'auteur.

38 Euripide, Hippolyte 1236-1247 "Cependant l'infortuné, embarrassé dans les rênes, sans pouvoir se dégager de ces liens funestes, était traîné à travers les rochers, qui lui brisaient la tête et déchiraient son corps [...] Enfin les rênes se brisent, je ne sais comment ; dégagé de ses liens, il tombe, près de rendre le dernier soupir. À l'instant les chevaux ont disparu $[\ldots] »$.

39 Voir à ce propos, M. PAschalis, "The Bull and the Horse : Animal Theme and Imagery in Seneca's Phaedra ", American Journal of Philology 115 (1994), p. 105-128. 
Dans le chapitre I de la deuxième partie, la maitresse d'auberge du Lion d'Or Madame Lefrançois demande à la servante de dire à Polyte, comme elle appelle affectueusement le garçon d'écurie, de déplacer une charrette, pour qu'elle ne soit pas détruite par la voiture Hirondelle qui va arriver. "Polyte " est un diminutif d'« Hippolyte " et apparaît seulement ici dans le roman (dans les manuscrits il apparait aussi ailleurs). Le lecteur n'a pas encore entendu le nom " Hippolyte ", alors il ne peut pas savoir qu'il s'agit d'un diminutif et par conséquent il est incapable d'établir une liaison entre le nom et le héros du roman. Dans le chapitre II de la deuxième partie, le garçon d'écurie accompagne les Bovary chez eux, une lanterne à une main, pour éclairer le chemin, et le parapluie de l'abbé Bournisien à l'autre. Son nom n'est pas mentionné, seulement sa qualité de "garçon d'écurie ». Le narrateur ajoute : "Sa chevelure rouge était entremêlée de brins de paille et il boitait de la jambe gauche ${ }^{40}$. " Le lecteur n'est pas suffisamment préparé pour identifier le personnage décrit ici avec Polyte.

Le prénom complet du garçon d'écurie apparaît dans le chapitre VIII de la deuxième partie :

« Hippolyte, le garçon de l'auberge, vint prendre par la bride les chevaux du cocher, et tout en boitant de son pied bot, il les conduisit sous le porche du Lion d'or, où beaucoup de paysans s'amassèrent à regarder la voiture ${ }^{41}$. "

La première différence évidente entre le passage cité ci-dessus et les précédents où le garçon d'écurie est présent est la liaison thématique et sémantique de son nom avec les chevaux. À l'opposition du nom "Polyte ", le nom " Hippolyte " s'inscrit dans un contexte sémantique qui met en valeur autant le composant ÏtாTos que le composant $\lambda u ́ \omega$. Dans un champ sémantique la combinatoire " Hippolyte " "bride " " chevaux " " cocher " met en valeur le composant İாTos du nom propre. Le deuxième composant ressortit aux activités d'Hippolyte en tant que garçon d'écurie : il vient prendre par la bride les chevaux des cochers, il les attelle et les dételle, il prend soin d'eux. La conduite des chevaux et de la voiture sous le porche de l'auberge du Lion d'Or évoque au lecteur l'encadrement du nom d'Hippolytos mythique par les verbes "attacher " et " détacher ${ }^{42}$ ". Contrairement à l'Hippolytos mythique, Hippolyte n'est pas un cavalier, ni un conducteur de char, mais un simple garçon d'écurie - le lecteur doit toujours avoir à l'esprit l'intention du narrateur de renverser le stéréotype du héros mythique qui est mentionné dans le lemme du Dictionnaire des idées reçues.

Le lecteur doit avoir déjà identifié Hippolyte avec le garçon boiteux qui avait accompagné les Bovary à leur nouveau domicile. Son problème est décrit comme " pied-bot » et on va voir un peu plus bas (unité 4) qu'il est étroitement lié au nom du

40 Flaubert, Euvres complètes, t. 1, p. 599 et 603.

41 Flaubert, Euvres complètes, t. 1, p. 622.

42 Dans les manuscrits, les chevaux et la voiture (de laquelle on apprend qu' elle " resta dételée ") continuent leurs trajets séparément (brouillons, vol. 3, folio 126). 
héros (Hippolyte) et à sa qualité (garçon d'écurie). Pour le moment on doit conserver à l'esprit que le prénom complet d'Hippolyte apparaît quand il vient prendre les chevaux du char du Conseiller "Lieuvain " (un nom qui renvoie au lieu vain et souligne ainsi de manière éloquente la vanité du personnage ${ }^{43}$ ) pour les conduire sous le porche de l'auberge du Lion d'Or. La référence à Hippolyte s'insère dans le contexte thématique d'un chapitre entier, dans lequel la présence et surtout la parole du Conseiller constituent des parties importantes. Le chapitre VIII de la deuxième partie est consacrée aux comices, que Flaubert décrit à Louise Colet comme une symphonie, où les beuglements de taureaux se rencontrent avec les soupirs d'amour (il s'agit de la séduction d'Emma Bovary par Rodolphe) et la rhétorique vide et pompeuse du représentant administratif (Lieuvain) :

"Si jamais les effets d'une symphonie ont été reportés dans un livre, ce sera là. Il faut que ça hurle par l'ensemble, qu'on entende à la fois des beuglements de taureaux, des soupirs d'amour et des phrases d'administrateurs ${ }^{44}$."

Dans ce chapitre la présence des animaux est la plus intense dans le roman. Dans un endroit clôturé sont rassemblées toutes les espèces d'animaux (bovins, porcs, chevaux, moutons), pour que les juges les voient, choisissent les meilleurs et leur décernent des prix. La description des animaux, leur sélection et leurs récompenses se croisent avec les descriptions des réunions des hommes et des femmes qui sont tous bien habillés pour l'occasion, ainsi qu'avec les distinctions d'honneur de la hiérarchie sociale. La rhétorique vide, publique, du Conseiller et la rhétorique banale, privée, de Rodolphe qui essaie de séduire Emma se rencontrent et exercent un charme sur leurs auditoires respectifs. Le fond sonore de cette scène est formé par les mugissements et les bêlements des animaux que les bergers ont conduits plus près de l'endroit où la harangue a lieu, dans leur effort pour mieux entendre, ainsi que par l'annonce des prix d'engrais, de progrès agricoles et d'élevage des animaux faite par le président du comité. Hommes et animaux, l'élément le plus haut et le plus bas de l'échelle sociale, sont devenus un : Rodolphe foule avec ces bottines chères et bien vernies les crottins de cheval (" elles étaient si vernies que l'herbe s'y reflétait ${ }^{45}$ »). Dans cet entourage Flaubert s'est proposé de réunir et de mettre en contact tous les personnages de son roman, comme il le commente lui-même de manière caractéristique dans une autre lettre adressée à Louise Colet (datée le 7 septembre 1853) : "J'y ai tous mes personnages de mon livre en action et en dialogue, les uns mêlés aux autres et par là-dessus un grand paysage qui les enveloppe ${ }^{46} .[\ldots] »$

43 Voir Porter et Gray, Gustave Flaubert's Madame Bovary, p. 104.

44 Flaubert, Correspondance, p. 449.

45 Flaubert, Euvres complètes, t. 1, p. 621.

46 Flaubert, Correspondance, p. 426 et plus en détail, p. 386 (lettre à Louise Colet datée du 7 juillet). 
La présence dans ce chapitre du garçon de l'auberge du Lion d'Or trouve ainsi son explication. Plus spécifiquement, l'apparition d'Hippolyte coopère avec la réunion et la présence intense de toute espèce animale, qui met en valeur le sens du composant ímாо et sa qualité de garçon d'écurie, comme l'a déjà remarqué $M$. Church ${ }^{47}$. Cette fonction complexe ainsi que l'étymologie du nom "Hippolyte " est en quelque sorte préfigurée dans la description paradoxale de l'endroit clôturé où les animaux sont rassemblés comme "une manière d'hippodrome ${ }^{48}$ ". En plus, dans un espace de quelques lignes, le nom "Hippolyte " est encadré par d'autres noms qui évoquent des animaux : le maire Tuvache est mentionné avant Hippolyte et Mme Tuvache est aussi mentionnée après l'auberge du Lion d'Or. Dans la suite de mon analyse je vais montrer que l'élément bestial constitue un trait fondamental d'Hippolyte. Dans la "symphonie " (selon les termes de Flaubert) du chapitre des comices, Hippolyte reste silencieux, contrairement aux animaux qui grognent, mugissent, ou bêlent et aux autres personnages qui prononcent des discours avec fatuité; nous allons l'entendre plus tard, après l'opération ratée de son pied, tout d'abord palpiter à cause de la douleur, ensuite supplier et enfin émettre le hurlement déchirant d'un animal égorgé.

\section{Le pied-bot d'Hippolyte}

Comme nous l'avons noté, le sens du nom "Hippolyte " est mis en valeur non seulement par sa liaison avec le cheval et en général les animaux du chapitre VIII de la deuxième partie, mais aussi par la première référence à son pied-bot. Les lettres de Flaubert à Louise Colet datées d'avril 1854 nous informent que pour l'écriture du chapitre XI de la deuxième partie sur l'opération du pied d'Hippolyte, Flaubert a consacré beaucoup de temps à l'étude du diagnostic et de la guérison des pieds bots et qu'il a littéralement dévoré le traité de Duval mentionné ci-dessus et intitulé Traité pratique du pied-bot. À ce propos, il a aussi demandé le secours de son frère Achille qui était chef des chirurgiens à l'hôpital de Rouen ${ }^{49}$ et avait succédé ainsi à leur père qui

47 Voir Church, Structure and Theme, p. 71 : «Pigs, calves, cows, rearing stallions, mares are lined up to be judged by a group of gentlemen who advance with heavy step. Horses and Hippolyte (a name derived from the Greek word for horse) go off together toward the stables "; et cf. aussi, p. 74 (après l'opération) : "The operation and the ensuing amputation also symbolically indicate the excision of the thematic concern with animals in part two ".

48 Flaubert, Euvres complètes, t. 1, p. 620.

49 Flaubert, Correspondance, t. 2, p. 544, 551, 556. Le chapitre XI de la deuxième partie présente une dépendance étroite avec le traité de Duval que Flaubert a " hérité " de la bibliothèque de son père ; voir EMPTAZ, "Gustave Flaubert apprenti orthopédiste ", p. 221-235. 
avait tenté sans succès de guérir un cas de pied-bot que Duval avait finalement guéri ${ }^{50}$. Le diagnostic qui a lieu avant l'opération du pied d'Hippolyte conclut qu'il s'agit d'un cas d'équin (talipes equinus) mêlé d'un peu de varus (talipes varus), finalement d'un léger varus fortement accusé d'équin (talipes equinovarus) :

"Cependant, pour savoir quel tendon couper à Hippolyte, il fallait connaître d'abord quelle espèce de pied-bot il avait. Il avait un pied faisant avec la jambe une ligne presque droite, ce qui ne l'empêchait pas d'être tourné en dedans, de sorte que c'était un équin mêlé d'un peu de varus, ou bien un léger varus fortement accusé d'équin. Mais, avec cet équin, large en effet comme un pied de cheval, à peau rugueuse, à tendons secs, à gros orteils, et où les ongles noirs figuraient les clous d'un fer, le stréphopode, depuis le matin jusqu'à la nuit, galopait comme un cerf. On le voyait continuellement sur la place, sautiller tout autour des charrettes, en jetant en avant son support inégal. Il semblait même plus vigoureux de cette jambe-là que de l'autre. À force d'avoir servi, elle avait contracté comme des qualités morales de patience et d'énergie, et quand on lui donnait quelque gros ouvrage, il s'écorait dessus, préférablement. Or, puisque c'était un équin, il fallait couper le tendon d'Achille $^{51}[\ldots]$ ».

Il faut noter d'abord que, parmi les cas divers de varus que Flaubert a trouvés dans le traité de Duval, il a choisi celui qui présente un rapport étymologique avec le nom et la qualité d'Hippolyte, à savoir le pied-bot équin ${ }^{52}$. En plus, bien que Duval distingue des cas qui sont " demi-équin et demi-varus ${ }^{53}$ ", Flaubert met l'accent sur le pied-bot équin et attribue seulement un rôle complémentaire au varus.

L'intention transparente de l'auteur de privilégier le pied-bot équin est réalisée au niveau sémantique. Dans le passage cité ci-dessus, le nom " Hippolyte " libère le sens

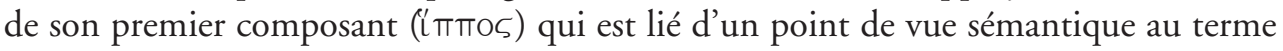

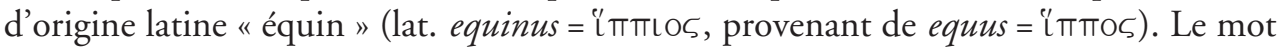
français « cheval " revient, mais cette fois à travers une description d'un membre du corps humain qui ressemble à un sabot. Or, bien que d'un point de vue sémantique les termes " équin " et " cheval " mettent en valeur le sens du mot iाmாо du premier composant du nom "Hippolyte ", du point de vue de la genèse narrative du terme pied-bot équin, c'est un processus inversé qui est suivi : l'invention du pied-bot équin et de l'image du pied à laquelle il renvoie est due au nom « Hippolyte ». Il faut ajouter ceci : il apparaît que Flaubert s'est rendu compte progressivement de l'importance de

50 Il a été proposé que l'opération ratée du pied d'Hippolyte constitue une critique indirecte de l'échec du père de Flaubert, mais les choses sont plus complexes; voir Brown, Flaubert, p. 309.

51 Flaubert, Euvres complètes, t. 1, p. 633-634.

52 EmptaZ, "Gustave Flaubert apprenti orthopédiste », p. 228-229.

53 Duval, Traité pratique, p. 15. 
son invention, puisque la description du pied comme un sabot n'apparaît pas dans les dessins antérieurs de ce passage.

En revanche, l'incision du tendon d'Achille et l'amputation du pied mettent en valeur le sens $\lambda \dot{v} \omega$ du deuxième composant du nom "Hippolyte ". Notons dans ce cas que la Phèdre de Sénèque se clôt avec une scène macabre, au cours de laquelle le

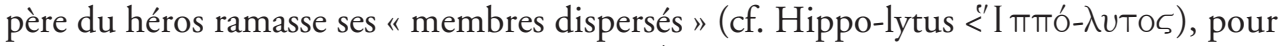
restituer son corps et le livrer intact au feu ${ }^{54}$. D'une manière analogue, dans Madame Bovary, Emma achète pour Hippolyte une jambe mécanique très chère et plus tard une deuxième pour un usage quotidien d'après le souhait d'Hippolyte qui " n'osant pas à tous les jours se servir d'une si belle jambe, supplia Madame Bovary de lui en procurer une autre plus commode ${ }^{55}$ ». La file sémantique " attacher/détacher ", qui est liée au composant $\lambda u ́ \omega$, est ainsi employée de manière créative.

Flaubert, en ayant recours à ces techniques littéraires, est arrivé ainsi à " battre " le récit stéréotypé de la mort d'Hippolytos, sur lequel il ironise dans le Dictionnaire des idées reçues. Il a créé un Hippolyte anti-conventionnel, un simple garçon d'écurie qui souffre de pied-bot, dont le pied ressemble à un sabot de cheval et qui est utilisé comme un cobaye pour qu'on lui applique une méthode chirurgicale catastrophique ${ }^{56}$.

L'essence de l'histoire est résumée dans la phrase "il galopait comme un cerf ", qui suggère une rencontre oxymorique des traits du cheval et des traits du cerf ${ }^{57}$. Hippolyte ressemble à un cheval, mais en réalité il est un cerf, voire une proie potentielle. Le pied du cheval peut lui procurer la vitesse et l'énergie d'un cheval, mais en même temps il le marginalise, il provoque l'opération chirurgicale et enfin l'amputation, au cours de laquelle Hippolyte est comparé à un animal égorgé. Chez Euripide et chez Sénèque, la chasse est l'activité primaire d'Hippolytos qu'il pratique accompagné par Artémis ${ }^{58}$. Il s'agit d'un exemple classique d'un héros ancien qui commence à agir comme un chasseur et finit par être lui-même la proie ${ }^{59}$. Les chevaux accompagnent le héros, mais

54 Sénèque, Phèdre 1247-1280.

55 Flaubert, Euvres complètes, t. 1, p. 638.

56 Cf. Church, Structure and Theme, p. 74 : "This operation symbolically injures the very root of his existence, for to the horse the leg means survival, and he is destroyed when his legs no longer perform."

57 P. PARLej, The Romantic Theory of the Novel: Genre and reflection in Cervantes, Melville, Flaubert, Joyce, and Kafka, Bâton rouge / London, 1997, p. 165-173, traite la description du pied d'Hippolyte comme un cas de bricolage d'auteur. Or, dans la mesure où il s'agit de la maladie d'Hippolyte (demi-équin et demi-varus), Duval, que Flaubert a consulté, mentionne qu'il s'agit toujours d'une combinaison de deux anomalies.

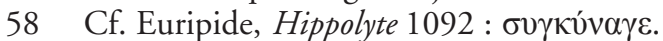

59 L'image finale du taureau féroce qui chasse ses chevaux en provoquant leur panique et par conséquent leur destruction (Euripide, Hippolyte 1218-1235 ; Sénèque, Phèdre 1077 1089) renverse l'image initiale du héros qui, comme un autre Héraclès, libère la terre des bêtes sauvages (Euripide, Hippolyte 17-18). Pour la métaphore de la chasse chez Hippolyte et ses riches connotations, voir C.P. SEGAL, "The tragedy of the Hippolytus : The waters 
ce sont eux qui provoquent à la fin sa mise en lambeaux, lorsque, paniqués par l'apparition du taureau féroce, ils le tirent sur les rochers (chez Sénèque, en particulier, le comportement des chevaux apparaît délibéré) ${ }^{60}$. Le rapport avec le cheval s'avère donc fatal autant pour Hippolytos que pour Hippolyte, de sorte que le sort d'Hippolyte peut être interprété comme une écriture moderne de la fortune d'Hippolytos ancien.

Nous pouvons maintenant examiner de plus près la comparaison d'Hippolyte avec le cerf. Chez Euripide le cerf est mentionné dans le premier acte comme une proie désirée par Phèdre ${ }^{61}:$ l'héroïne fait sa première apparition dans un état de passion amoureuse et en cherchant les eaux pures et les prairies riches, à savoir les lieux fréquentés par Hippolytos; elle désire aller chasser avec les chiens des cerfs mouchetés (Ba入ıaĩs $\left.\dot{\epsilon}^{\prime} \lambda a ́ \phi o ı s\right)^{62}$ dans les montagnes boisées ${ }^{63}$. Vue la liaison entre Emma et Phèdre proposée auparavant en raison de leur rôle respectif dans la destruction d'Hippolyte et d'Hippolytos, le passage d'Euripide doit probablement être pris en considération pour l'interprétation de la comparaison d'Hippolyte avec un cerf.

La gangrène conduira finalement à la convocation du médecin Canivet, qui décide immédiatement d'amputer Hippolyte - Charles n'était point autorisé à faire cette opération, parce qu'il était un simple "officier de santé ». Canivet est un personnage corpulent qui s'intéresse plus à soigner son cheval que ses patients (quelle ironie pour le malheureux Hippolyte!) ; il déclare cyniquement : «il m’est aussi parfaitement égal de découper un chrétien que la première volaille venue " et se réjouit lorsque le pharmacien compare "le sang-froid d'un chirurgien à celui d'un général ${ }^{64}$ ". Il dénonce ceux qui ont fait l'opération (" est-ce qu'on peut redresser les pieds bots ? C'est comme si l'on voulait, par exemple, rendre droit un bossu $\left.{ }^{65} ! »\right)$ et sauve la vie d'Hippolyte, mais il provoque plus tard la mort d'Emma en se trompant de médicament ${ }^{66}$.

of the ocean and the untouched meadow ", In memoriam Arthur Darby Nock, Harvard Studies in Classical Philology 70 (1965), p. 117-169.

60 Voir Paschalis, «The Bull and the Horse », p. 117-118.

61 Chez Sénèque il est mentionné comme une proie d'Artémis (Phèdre 61-63).

62 Euripide, Hippolyte 218-221.

63 Racine donne une version raccourcie de ce passage en omettant la chasse, mais toutes les éditions commentées de sa tragédie donnaient les vers d'Euripide (au cas où Flaubert n'avait pas devant les yeux la tragédie d'Euripide). Je donne à titre d'exemple une édition antérieure et une autre postérieure au temps d'écriture de Madame Bovary: M. LUNEAU De Boisjermain, Commentaires sur les auvres de Jean Racine, t. 3, Paris, 1768, p. 57-58; M.L. Moland, Euvres completes de J. Racine avec une vie de l'auteur et un examen de chacun de ses ouvrages, t. 4, Paris, 1875, p. 303.

64 Flaubert, Euvres complètes, t. 1, p. 636.

65 Ibid.

66 Pour la responsabilité de Canivet de la mort d'Emma, voir L. Rothfield, Medical Signs : Medical Realism in Nineteenth-Century Fiction, Princeton, 1992, p. 18. 
Le cynisme avec lequel il se sert de la lame chirurgicale est résumé de manière ironique et dépréciative dans le nom "Canivet" qui évoque le canif ${ }^{67}$.

La procédure d'amputation dure assez longtemps et le lecteur participe à cette scène à travers les réflexions de Charles et d'Emma (au niveau sémantique, le lecteur attentif remarque l'encadrement du nom "Hippolyte " par le nom "Bovary "). Tous les deux conçoivent la fortune du malheureux garçon d'écurie du point de vue de leur intérêt personnel : le premier songe aux conséquences de son échec, tandis que la deuxième éprouve du dédain pour son mari. Leurs pensées sont interrompues à deux reprises par la souffrance de l'amputé : la première fois " un cri déchirant traversa l'air " et la deuxième fois les cris continus d'Hippolyte sont décrits comme "un hurlement lointain de quelque bête qu'on égorge ${ }^{68} \%$. La comparaison souligne la nature bestiale d'Hippolyte et en même temps suggère que la lame tranchante du médecin Canivet morcelle sans distinction hommes et animaux.

Les apparitions d'Hippolyte après l'opération ratée de son pied soulignent emphatiquement son handicap, qui entraîne pour lui une mobilité pénible et pour Charles une évocation embarrassante de son incompétence. Le galop rapide du pied de cheval est maintenant remplacé par le son constamment lent et sec de la jambe artificielle en bois.

Hippolyte réapparaît en tant que figure misérable devant Emma et Charles le jour où celle-ci apprend la mort de son beau-père, et pour la dernière fois à l'église où ont lieu les funérailles d'Emma. Contrairement aux mythes des tragédies qui ont comme sujet la fortune d'Hippolytos, il n'y a ici aucun repentir, aucune lamentation, seulement la gêne de Charles qui néanmoins n'éprouve aucune sympathie pour sa victime. Emma se suicide tout comme Phèdre, mais pour des raisons qui n'ont rien à voir avec la fortune du malheureux Hippolyte. Les deux passages suivants sont révélateurs :

a) «Ils entendirent dans le vestibule le bruit sec d'un bâton sur les planches. C'était Hippolyte qui apportait les bagages de Madame. Pour les déposer, il décrivit péniblement un quart de cercle avec son pilon.

- Il n'y pense même plus! se disait-elle en regardant le pauvre diable, dont la grosse chevelure rouge dégouttait de sueur.

Bovary cherchait un patard au fond de sa bourse ; et, sans paraitre comprendre tout ce qu'il y avait pour lui d'humiliation dans la seule présence de cet homme qui se tenait là, comme le reproche personnifié de son incurable ineptie :

- Tiens! tu as un joli bouquet! dit-il en remarquant sur la cheminée des violettes de Léon ${ }^{69}$ ".

67 Porter et Gray, Gustave Flaubert's Madame Bovary, p. 104.

68 Flaubert, Euvres complètes, t. 1, p. 637.

69 Flaubert, Euvres complètes, t. 1, p. 659. L'attitude de Charles révèle une gêne ; cf. aussi, Flaubert, Euvres complètes, t. 1, p. 638, où il prend une autre route, quand il entend de loin le bruit du bâton d'Hippolyte. Flaubert a pris soin de dissimuler cette gêne assez bien : dans une version antérieure (brouillons, vol. 5, folio 170v), Charles attend 
b) «On entendit sur les dalles comme le bruit sec d'un bâton ferré qui les frappait à temps égaux. Cela venait du fond, et s'arrêta court dans les bas-côtés de l'église. Un homme en grosse veste brune s'agenouilla péniblement. C'était Hippolyte, le garçon du Lion d'or. Il avait mis sa jambe neuve.

L'un des chantres vint faire le tour de la nef pour quêter, et les gros sous, l'un après l'autre, sonnaient dans le plat d'argent.

- Dépêchez-vous donc! je souffre, moi ! s'écria Bovary, tout en lui jetant avec colère une pièce de cinq francs. L'homme d'église le remercia par une longue révérence ${ }^{70}$. "

Dans un brouillon du roman Flaubert présente ainsi la dernière apparition d'Hippolyte :
« Hippolyte doit
paraître à l'enterrement
bruit du pilon $-\&$
balancement, comme
un oiseau blessé ${ }^{71}$."

Flaubert a finalement supprimé la comparaison d'Hippolyte avec un oiseau blessé. Ce détail témoignait d'une affection, mais avait aussi une fonction plus importante : il soulignait le contraste entre l'état antérieur du garçon d'écurie et son état après l'opération, voire le contraste entre la liberté d'antan et le handicap du présent. En revanche, dans la version définitive du roman, c'est Emma qui est constamment comparée à un oiseau $^{72}$. La comparaison la plus révélatrice apparaît quand tout est perdu et la fin de l'héroïne approche : seule, fatiguée et dégoûtée, enfermée dans une chambre de l'Hôtel de Boulogne de Rouen, Emma est possédée par un désir ardent de voler comme un oiseau : "Elle aurait voulu, s'échappant comme un oiseau, aller se rajeunir quelque part, bien loin, dans les espaces immaculés ${ }^{73}$. " Hippolyte ne sait pas que c'est Emma qui a convaincu Charles de lui faire l'opération. Il assiste à ses funérailles, parce qu'il ressent de la gratitude envers elle à cause de la jambe artificielle qu' elle lui a offerte. La comparaison de ces deux personnages avec des oiseaux qui ne peuvent pas voler suggère une rencontre profonde au niveau humain. Flaubert a bien sûr supprimé la comparai-

qu'Hippolyte s'éloigne, il marche nerveusement pour quelques minutes et fait ensuite une remarque sur les violettes de Léon. Notons enfin que dans un autre brouillon (brouillons, vol. 5, folio 112v), Hippolyte s'approche pour recevoir un pourboire. Il semble que dans la version finale Flaubert a voulu insister sur le fait que Charles a gardé sa dignité.

70 Flaubert, Euvres complètes, t. 1, p. 688.

71 Brouillons, vol. 5, folio 1.

72 Euvres complètes, t. 1, p. 610 : «sa démarche d'oiseau »; p. 611 : «Alors un attendrissement la saisit, elle se sentit molle et tout abandonnée, comme un duvet d'oiseau qui tournoie dans la tempête "; p. 655 : "Elle répondit par un signe de tête et disparut comme un oiseau dans la pièce à côté" ".

73 Euvres complètes, t. 1, p. 673. 
son avec l'oiseau pour Hippolyte, mais la conclusion à tirer reste la même : Emma a toujours été handicapée ; Hippolyte au début ne l'était pas, mais il a fini par le devenir à cause des Bovary, à cause de la vanité d'Emma et de l'incompétence de Charles. Dans une étude très intéressante, F. Emptaz a récemment lié le handicap d'Hippolyte au handicap métaphorique d'Emma Bovary en soulignant avec justesse le rapport entre les aventures amoureuses d'Emma et les apparitions d'Hippolyte ; elle a été fondée sur une proposition antérieure de Jean Maurel, selon laquelle le varus d'Hippolyte se reflète dans le nom "Bovary ", de sorte que l'état boiteux est inscrit dans le nom de l'héroïne : "Madame Beau (pied bot) varus ${ }^{74}$."

Je voudrais conclure cette unité en discutant le nom de famille d'Hippolyte qui dans le roman est mentionné une seule fois. À la suite de l'opération le pharmacien Homais envoie une annonce au journal le Fanal de Rouen, pour célébrer la réussite de Charles et pour augmenter sa propre publicité. Dans ce passage le nom complet d'Hippolyte apparaît en combinaison avec d'autres éléments concernant ce personnage : "M. Bovary, un de nos praticiens les plus distingués, a opéré d'un pied-bot le nommé Hippolyte Tautain, garçon d'écurie depuis vingt-cinq ans à l'hôtel du Lion d'or, tenu par Mme veuve Lefrançois, sur la Place d'armes ${ }^{75}$."

Une étymologie proposée pour le nom «Tautain » est qu'il s'agit d'un cas d'onomatopée : le nom évoque le son " tautain-tautain-tautain " que les enfants produisent avec leurs langues pour imiter les sabots du cheval et en même temps renvoie à la marche du garçon d'écurie boiteux ${ }^{76}$. Dans cette perspective, Demorest avait aussi observé, comme nous l'avons noté auparavant, que le prénom " Hippolyte » du héros évoque sa marche sautillante. Notons aussi que dans un des manuscrits ${ }^{77}$ Flaubert emploie l'expression fort acoustique " clopin-clopant » pour décrire la marche boiteuse d'Hippolyte.

Néanmoins, une étymologie plus féconde peut aussi être avancée. Dans un idiome de Normandie, patrie de Flaubert et lieu où se déroule le roman, on trouve le mot " tautaus " qui signifie " gros sabots ». Cette information est fournie par un dictionnaire qui a paru trois ans avant le début de l'écriture de Madame Bovary ${ }^{78}$. Le mot sabot signifie à la fois l'onglon (d'un cheval ou d'un autre animal) et la chaussure

74 F. Emptaz, Aux pieds de Flaubert, Paris, 2002, p. 25 ; J. Maurel, "Une dame bête comme ses pieds : le coup de patte de Flaubert ", Revue des sciences humaines 181 (1981), Numéro spécial consacré à Gustave Flaubert, p. 108.

75 Flaubert, Euvres complètes, t. 1, p. 634. La présentation des éléments qui concernent l'identité d'Hippolyte est en partie dictée par le modèle du traité de Duval suivi par Flaubert ; voir EMPTAZ, « Gustave Flaubert apprenti orthopédiste », p. 231.

76 EMPTAZ, «Gustave Flaubert apprenti orthopédiste », p. 229.

77 Brouillons, vol. 3, folio 126.

78 M.M. Édélestand, Alfred Duméril, Dictionnaire du patois Normand, Caen, 1849, p. 204. Il s'agit de l'idiome de la Basse-Normandie et non de la Haute-Normandie, patrie de Flaubert, mais cet élément semble secondaire pour un roman. 
en bois : il combine ainsi idéalement l'état précédent (pied comme un sabot d'un cheval) ${ }^{79}$ et l'état suivant (pied en bois) d'Hippolyte.

Enfin, le lecteur doit aussi considérer que le nom "Hippolyte Tautain " évoque le nom du philosophe, historien et critique français Hippolyte Taine. La coïncidence acoustique est si grande qu'elle passerait difficilement inaperçue. Flaubert appréciait beaucoup Taine et plus tard ils ont développé des liens d'amitié. Cependant, il semble que les deux hommes ne s'étaient pas encore rencontrés pendant la période de l'écriture de Madame Bovary (1851-1856). L'identification de ces deux Hippolyte semble difficile à vérifier. Peut-être s'agit-il d'une plaisanterie, d'un jeu de mots qui ne révèle pas d'intention concrète ${ }^{80}$.

\section{D’Hippolytos à Hippolyte : remarques finales}

Quelle est alors l'identité d'Hippolyte ? Le récit du chapitre XI de la deuxième partie sur l'opération chirurgicale de son pied est significatif pour sa présence dans le roman. Il a l'air d'un diagnostic clinique sorti du traité de Duval. En se fondant sur cet ouvrage, Flaubert décrit avec une précision scientifique un membre du corps d'Hippolyte. Dans le passage cité plus haut, la perspective de Charles se croise avec celle du narrateur ${ }^{81}$. L'état misérable de la jambe d'Hippolyte après l'opération est décrit avec la même précision (œdème, ecchymoses, convulsions, douleur torturante et enfin gangrène).

On pourrait bien prétendre que le cas du pied d'Hippolyte est paradigmatique du réalisme dans Madame Bovary, un roman dans lequel le récit met l'accent plutôt sur les parties que sur l'ensemble ${ }^{82}$. Dans ce cas, la partie dispose de traits bestiaux, à savoir d'un pied qui ressemble à un sabot de cheval et avec lequel le héros galope comme un cerf. Il y a néanmoins un rapport entre la partie et l'ensemble, puisque le nom " Hippolyte " évoque le mot " cheval » et puisque lui-même prend soin des chevaux des visiteurs de l'auberge; de plus, pendant l'amputation, il crie comme « un animal qu'on égorge ».

79 Notons aussi que dans le passage que nous venons de citer Flaubert décrit le pied d'Hippolyte ainsi : "large en effet comme un pied de cheval ".

80 Dans Madame Bovary il y a aussi un autre jeu de mots entre un héros et un personnage historique, mais dans ce cas la liaison proposée est transparente. Flaubert a nommé le sacristain, fossoyeur et bedeau d'Yonville Lestiboudois, évidemment pour suggérer une liaison avec le botaniste Jean-Baptiste Lestiboudois (1715-1804), qui en 1737 a écrit un traité pour louer les qualités alimentaires de la pomme de terre et les bénéfices qu'on peut en tirer si on la cultive.

81 Pour la difficulté de distinguer la voix du narrateur de celle d'un personnage du roman chez Flaubert, voir J. Culler, "The Real Madame Bovary », in B. Vinken, P. Fröhlicher (éd.), Le Flaubert réel, Tübingen, 2009, p. 9-20.

82 Voir P. BRooks, Realist Vision, New Haven / London, 2005, p. 54-70. 
On apprend les pensées, les émotions et les paroles d'Hippolyte seulement avant, pendant et après l'opération au tendon de son pied. Il y a d'abord la pression que le pharmacien Homais exerce sur lui, afin de le persuader de donner son consentement pour l'opération. Quand Homais le rassure en affirmant que la douleur sera légère (" une simple piqûre comme une petite saignée »), Hippolyte réagit ainsi : " réfléchissant, roulait ses yeux stupides ". À l'argument du pharmacien selon lequel après l'opération "il s'en trouverait mieux pour plaire aux femmes " Hippolyte "se prenait à sourire lourdement ${ }^{83}$ ». La rapidité de l'opération le surprend tant qu'il « se penchait sur les mains de Bovary pour les couvrir de baisers ${ }^{84}$ ". Après l'opération on apprend les convulsions de son pied et la douleur qui progressivement devient torturante, mais on entend sa voix seulement une fois, quand il ne peut plus la supporter. Il balbutie en sanglotant et prie Charles Bovary de le sauver : "Quand est-ce que je serai guéri ?... Ah, sauvez-moi !... Que je suis malheureux! Que je suis malheureux ${ }^{85}$ ! " Afin qu'il se libère de son martyre, Hippolyte promet à l'abbé Bournisien de réciter matin et soir un " Je vous salue Marie, pleine de grâce " et un " Notre Père, qui êtes aux cieux "; il promet en plus d'aller en pèlerinage à Bon Secours, s'il guérit. Le narrateur commente aussi à ce propos qu'Hippolyte entendait mais "ne comprenait pas » les anecdotes et les plaisanteries que l'abbé racontait à l'aubergiste Mme Lefrançois ${ }^{86}$.

En résumé, Hippolyte dispose de traits et d'un nom bestiaux ; il se caractérise en plus par une intelligence très lente et une réaction instinctive à la douleur qui, au moment de son amputation, évoque une bête (notons à ce propos que "bête " signifie aussi stupide ${ }^{87}$ ). Comme nous l'avons noté auparavant, J. Culler, en jouant avec les mots « réalisme » (realism) et « veau » a parlé du "véalisme » de Madame Bovary; on pourrait parler en général de " bestialisme » en jouant avec les mots " bête " et " réalisme ". Mais les choses sont plus complexes quand il s'agit du réalisme de Flaubert ${ }^{88}$ et si on tient compte du niveau de lecture plus profond que suggère l'intertextualité. Ainsi, même les points qui semblent extrêmement réalistes peuvent évoquer des récits idéalistes ou romantiques. Par exemple, un commentateur a lié la torture de cet homme "bestial " à la torture que subissent les bêtes dans les autres œuvres de Flaubert ${ }^{89}$, tandis que

83 Flaubert, Euvres complètes, t. 1, p. 633.

84 Flaubert, Euvres complètes, t. 1, p. 634.

85 Flaubert, Euvres complètes, t. 1, p. 635.

86 Ibid.

87 Dans un des manuscrits (Mort du père Bovary-copiste, vol. 5, folio 107), Flaubert parle de la bêtise d'Hippolyte, une allusion qu'il a néanmoins ensuite supprimée.

88 Pour les problèmes du réalisme dans Madame Bovary, voir Culler, "The real Madame Bovary ".

89 R. Huss, "Nature, Final Causality and Anthropocentrism in Flaubert", French Studies 33 (1979), p. 288-304, sp., p. 295 : " Hippolyte is, indeed, closest of all the Yonvillais to the world of the animal so often tormented in other Flaubert texts ». L'auteur commente aussi la présence du mendiant aveugle du roman, dont les cris évoquent " un chien qui a faim ». 
d'autres ont constaté un rapport avec le martyre de Saint Hippolyte où, comme nous l'avons noté plus haut, le motif de la mise en lambeaux d'Hippolyte est aussi présent. Enfin, d'autres commentateurs ont comparé la boîte dans laquelle Charles ferme le pied d'Hippolyte à un instrument de torture qui évoque le brodequin qu'on a placé au pied d'Esmeralda dans Notre Dame de Paris de V. Hugo : et cela, parce que cet instrument de Charles est différent de ceux que décrivent les traités de l'époque ; en outre, l'influence générale du roman de $\mathrm{V}$. Hugo sur Madame Bovary est certaine (par exemple, le chien de chasse d'Emma s'appelle Djali, tout comme la petite chèvre d'Esmeralda) ${ }^{90}$.

Dans mon étude, j'ai tenté de montrer qu'Hippolyte tire son origine du héros mythique du même nom et que cette origine évolue et fonctionne à plusieurs niveaux. En effet, l'intertextualité crée des ruptures continues dans l'édifice du réalisme (ou du "bestialisme ") à travers lequel on peut reconnaitre le mythe ancien. Mais quel est le sens profond du recours au mythe ancien ? Le garçon d'écurie boiteux est-il inventé simplement comme une caricature du héros mythique d'origine royale, du cavalier et chasseur, compagnon de la déesse Artémis, qui est trainé par ses chevaux effrayés, jusqu'à ce que son corps se démembre?

Hippolytos et Hippolyte constituent, chacun pour des raisons différentes, des cas sociaux déviants et pour cela ils sont châtiés. L'Hippolytos d'Euripide est un homme misogyne sans amour qui accompagne la déesse Artémis à la chasse en méprisant Aphrodite. Aphrodite provoque la passion de Phèdre avec toutes les conséquences que nous venons d'analyser. En revanche, la particularité de l'Hippolyte de Flaubert réside dans sa faiblesse corporelle et non dans sa "pureté " (Brown), puisque, en raison de sa laideur, il est incapable d'inspirer une passion amoureuse ; il n'éprouve pas ce sentiment non plus, comme un autre Quasimodo (ici s'arrêtent les similitudes avec le héros de V. Hugo). Pourtant, malgré son pied-bot, il a l'agilité et la vitesse d'un cerf et selon le narrateur « il semblait même plus vigoureux de cette jambe-là que de l'autre [...] elle avait contracté comme des qualités morales de patience et d'énergie ${ }^{91}$ ». Hippolyte est content de lui-même et son comportement ne provoque pas la société et la mentalité prédominante, contrairement à l'attitude rigide et l'hybris de l'Hippolyte d'Euripide, et l'orgueil et la fierté de l'Hippolyte de Racine.

Or, bien que la particularité d'Hippolyte soit positivement appréhendée dans la perspective du narrateur, les protagonistes du roman n'acceptent pas son pied déformé et essaient de le redresser. La petite communauté d'Yonville exerce aussi une pression dans cette direction et s'imagine même qu' " Hippolyte allait reparaître marchant droit ${ }^{92}$ ".

90 Voir E. Zollinger, "Flaubert, Hugo, Ovide : la vengeance d'Arachné ", dans Le Flaubert réel, 47-58 (sp. p. 50-51) ; EMPTAZ, "Gustave Flaubert apprenti orthopédiste", p. 232-235 ; W. Adams, Club-foot : Its Causes, Pathology, and Treatment, London, 1866, p. $105-106$.

91 Flaubert, Euvres complètes, t. 1, p. 633-634.

92 Flaubert, Euvres complètes, t. 1, p. 634. 
À l'instar du mythe d'Hippolytos, la fin est tragique, puisque, à la suite de l'opération, Hippolyte devient vraiment handicapé : son pied tors mais habile est remplacé par un pied en bois droit mais qui entraîne pour lui une mobilité difficile et pénible.

En somme, une des raisons pour lesquelles Flaubert invente Hippolyte, le transforme en une victime et le torture en le rendant handicapé est pour se railler de l'absence de tolérance sociale à l'égard du handicap corporel du garçon d'écurie et surtout pour critiquer l'incompétence, la recherche du profit personnel, la vanité et la fatuité des protagonistes du roman, traits qui expliquent ensuite leur indifférence à la douleur qu'ils provoquent. Hippolyte est soumis à une opération pour des raisons soi-disant humanitaires, tout comme dans une autre société on se moquerait de son handicap.

Cette « conjuration » qui conduit Hippolyte à la destruction, reproduit dans la ville imaginaire du XIX $X^{e}$ siècle le scénario de la fortune de l'Hippolytos mythique, selon lequel Aphrodite, Phèdre, Thésée, Poséidon et ses auxiliaires " collaborent " pour faire du mal au jeune homme ${ }^{93}$. Chez Flaubert, l'inspirateur principal est le pharmacien Homais, un personnage sans scrupules (un autre nom ironique !) qui est présenté comme un partisan du progrès et lecteur des philosophes des Lumières; celui-ci met en scène l'opération, tout comme chez Euripide Aphrodite met en scène le châtiment d'Hippolytos, parce que ce dernier adore Artémis (Flaubert place une "Artémis » à côté d'Hippolyte dans le personnel de l'auberge du Lion d'Or) ${ }^{94}$. Les instruments d'Homais sont la vaniteuse Emma-Phèdre, l'incompétent et naïf Charles, qui est facilement convaincu par sa femme, tout comme Thésée est convaincu par les calomnies de Phèdre, et finalement la communauté entière d'Yonville. Le médecin cynique Canivet se sert efficacement de sa lame chirurgicale afin d'amputer le malheureux garçon d'écurie. La sympathie que le narrateur exprime envers Hippolyte crée un contraste patent avec l'indifférence qui caractérise les personnages du roman ; dans un des ses brouillons, Flaubert a fait preuve même d'une certaine affection, lorsqu'il a décrit Hippolyte comme " un oiseau blessé », en suggérant ainsi une similitude avec Emma. Mutatis mutandis, le cas d'Hippolyte évoque celui de la petite Berthe, qui, parce qu'elle est une enfant, a une conception insuffisante des choses et devient elle aussi la victime innocente de l'individualisme et des caprices de sa mère, elle devient orpheline de ses deux parents au cours du récit et finit par travailler comme ouvrière dans une usine d'égrenage de $\operatorname{coton}^{95}$.

En ce qui concerne la question initiale de savoir pourquoi Flaubert a retravaillé le récit de l'Hippolytos mythique, j'ai tenté de montrer que cela constitue un choix

93 Cf. l'interprétation symboliste quoique un peu confuse de M. Church, Structure and Theme, p. 74-75.

94 Il s'agit d'Artémise, servante à la même auberge. Son nom rend le nom grec "Artémisia " qui dérive du nom "Artémis " et bien sûr évoque le nom de la déesse.

95 Les commentateurs en général comparent la fortune d'Hippolyte avec celle du mendiant aveugle du roman ; cf. Huss, « Nature », p. 295. 
programmatique de l'auteur lié au lemme «Hippolyte » du Dictionnaire des idées reçues. Il y est noté avec un ton d'ironie que la mort d'Hippolytos est le plus beau sujet à raconter et que tout le monde doit le savoir par cœur. En me fondant sur une comparaison de la version définitive du roman avec les versions antérieures des manuscrits, j'ai proposé que le contenu du lemme a un rapport avec la genèse, la conception et la formation du héros du même nom : l'écrivain a cherché à proposer une alternative nouvelle au stéréotype banal du récit mythique.

Michael PASCHALIS

Professeur de lettres classiques

Université de Crète

51, rue Patelarou

G-74100 Réthymnon

michael.paschalis@gmail.com 